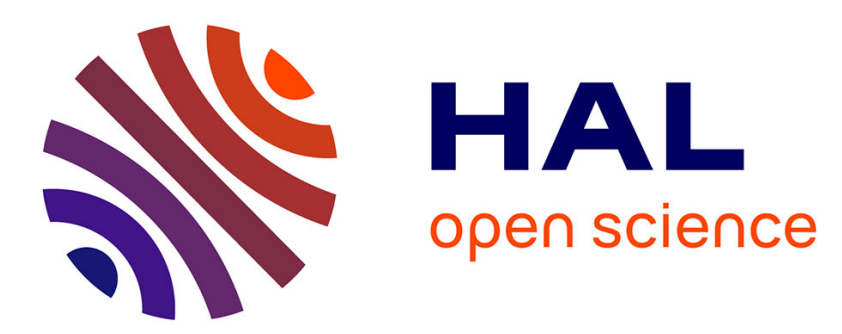

\title{
Algorithms of Robust Stochastic Optimization Based on Mirror Descent Method
}

Alexander V. Nazin, Arkadi S Nemirovsky, Alexandre B. Tsybakov, Anatoli B. Juditsky

\section{- To cite this version:}

Alexander V. Nazin, Arkadi S Nemirovsky, Alexandre B. Tsybakov, Anatoli B. Juditsky. Algorithms of Robust Stochastic Optimization Based on Mirror Descent Method. Automation and Remote Control / Avtomatika i Telemekhanika, 2019, 80 (9), pp.1607-1627. 10.1134/S0005117919090042 . hal02173931

\section{HAL Id: hal-02173931 \\ https://hal.science/hal-02173931}

Submitted on 4 Jul 2019

HAL is a multi-disciplinary open access archive for the deposit and dissemination of scientific research documents, whether they are published or not. The documents may come from teaching and research institutions in France or abroad, or from public or private research centers.
L'archive ouverte pluridisciplinaire HAL, est destinée au dépôt et à la diffusion de documents scientifiques de niveau recherche, publiés ou non, émanant des établissements d'enseignement et de recherche français ou étrangers, des laboratoires publics ou privés. 


\title{
Algorithms of Robust Stochastic Optimization Based on Mirror Descent Method
}

\author{
A.B. Juditsky, ${ }^{*} \quad$ A.V. Nazin,$^{\dagger} \quad$ A.S. Nemirovsky, ${ }^{\ddagger} \quad$ A.B. Tsybakov ${ }^{\S}$
}

\begin{abstract}
We propose an approach to the construction of robust non-Euclidean iterative algorithms by convex composite stochastic optimization based on truncation of stochastic gradients. For such algorithms, we establish sub-Gaussian confidence bounds under weak assumptions about the tails of the noise distribution in convex and strongly convex settings. Robust estimates of the accuracy of general stochastic algorithms are also proposed.
\end{abstract}

Keywords: robust iterative algorithms, stochastic optimization algorithms, convex composite stochastic optimization, mirror descent method, robust confidence sets.

\section{Introduction}

In this paper, we consider the problem of convex composite stochastic optimization:

$$
\min _{x \in X} F(x), \quad F(x)=\mathbf{E}\{\Phi(x, \omega)\}+\psi(x),
$$

where $X$ is a compact convex subset of a finite-dimensional real vector space $E$ with norm $\|\cdot\|, \omega$ is a random variable on a probability space $\Omega$ with distribution $P$, function $\psi$ is convex and continuous, and function $\Phi: X \times \Omega \rightarrow \mathbf{R}$. Suppose that the expectation

$$
\phi(x):=\mathbf{E}\{\Phi(x, \omega)\}=\int_{\Omega} \Phi(x, \omega) d P(\omega)
$$

is finite for all $x \in X$, and is a convex and differentiable function of $x$. Under these assumptions, the problem (1) has a solution with optimal value $F_{*}=\min _{x \in X} F(x)$.

Assume that there is an oracle, which for any input $(x, \omega) \in X \times \Omega$ returns a stochastic gradient that is a vector $G(x, \omega)$ satisfying

$$
\mathbf{E}\{G(x, \omega)\}=\nabla \phi(x) \quad \text { and } \quad \mathbf{E}\left\{\|G(x, \omega)-\nabla \phi(x)\|_{*}^{2}\right\} \leq \sigma^{2}, \quad \forall x \in X,
$$

\footnotetext{
${ }^{*}$ LJK, Université Grenoble Alpes, Grenoble anatoli.juditsky@univ-grenoble-alpes.fr

${ }^{\dagger}$ Institue of Control Sciences RAS, Moscow nazine@ipu.ru

${ }^{\ddagger}$ ISyE, Georgia Institute of Technology, Atlanta nemirovs@isye.gatech.edu

${ }^{\S}$ CREST, ENSAE alexandre.tsybakov@ensae.fr

Work of A.V. Nazin was supported by the Russian Science Foundation (Grant No. 16-11-10015), work of A.B. Tsybakov was supported by the GENES Institute and by the grant Labex Ecodec (ANR-11-LABEX-0047). A.B. Juditsky was supported by the grant PGMO 2016-2032H and A.B. Juditsky and A.S. Nemirovsky were supported by the NSF grant CCF-1523768.
} 
where $\|\cdot\|_{*}$ is conjugate norm to $\|\cdot\|$, and $\sigma>0$ is a constant. The aim of this paper is to construct $(1-\alpha)$-reliable approximate solutions of the problem (1), i.e., solutions $\widehat{x}_{N}$, based on $N$ queries of the oracle and satisfying the condition

$$
\operatorname{Prob}\left\{F\left(\widehat{x}_{N}\right)-F^{*} \leq \delta_{N}(\alpha)\right\} \geq 1-\alpha, \quad \forall \alpha \in(0,1),
$$

with as small as possible $\delta_{N}(\alpha)>0$.

Note that stochastic optimization problems of the form (1) arise in the context of penalized risk minimization, where the confidence bounds (3) are directly converted into confidence bounds for the accuracy of the obtained estimators. In this paper, the bounds $(3)$ are derived with $\delta_{N}(\alpha)$ of order $\sqrt{\ln (1 / \alpha) / N}$. Such bounds are often called sub-Gaussian confidence bounds. Standard results on subGaussian confidence bounds for stochastic optimization algorithms assume boundedness of exponential or subexponential moments of the stochastic noise of the oracle $G(x, \omega)-\nabla \phi(x)$ (cf. [1, 2, 3]). In the present paper, we propose robust stochastic algorithms that satisfy sub-Gaussian bounds of type (3) under a significantly less restrictive condition (2).

Recall that the notion of robustness of statistical decision procedures was introduced by J. Tukey [4] and P. Huber [5, 6, 7] in the 1960ies, which led to the subsequent development of robust stochastic approximation algorithms. In particular, in the 1970ies-1980ies, algorithms that are robust for wide classes of noise distributions were proposed for problems of stochastic optimization and parametric identification. Their asymptotic properties when the sample size increases have been well studied, see, for example, $[8,9,10,11,12,13,14,15,16]$ and references therein. An important contribution to the development of the robust approach was made by Ya.Z. Tsypkin. Thus, a significant place in the monographs $[17,18]$ is devoted to the study of iterative robust identification algorithms.

The interest in robust estimation resumed in the 2010ies due to the need to develop statistical procedures that are resistant to noise with heavy tails in high-dimensional problems. Some recent work [19, 20,21, 22, 23, 24, 25, 26, 27, 28, 29] develops the method of median of means [30] for constructing estimates that satisfy sub-Gaussian confidence bounds for noise with heavy tails. Thus, in [27] the median of means approach was used to construct an $(1-\alpha)$-reliable version of stochastic approximation with averaging ("batch" algorithm) in a stochastic optimization setting similar to (1). Other original approaches were developed in [31, 32, 33, 34, 35], in particular, the geometric median techniques for robust estimation of signals and covariance matrices with sub-Gaussian guarantees $[34,35]$. Also there was a renewal of interest in robust iterative algorithms. Thus, it was shown that robustness of stochastic approximation algorithms can be enhanced by using the geometric median of stochastic gradients [36, 37]. Another variant of the stochastic approximation procedure for calculating the geometric median was studied in [38, 39], where a specific property of the problem (boundedness of the stochastic gradients) allowed the authors to construct $(1-\alpha)$-reliable bounds under a very weak assumption about the tails of the noise distribution.

This paper discusses an approach to the construction of robust stochastic algorithms based on truncation of the stochastic gradients. It is shown that this method satisfies sub-Gaussian confidence bounds. In Sections 2 and 3, we define the main components of the optimization problem under consideration. In Section 4, we define the robust stochastic mirror descent algorithm and establish confidence bounds for it. Section 5 is devoted to robust accuracy estimates for general stochastic algorithms. Finally, Section 6 establishes robust confidence bounds for problems, in which $F$ has a quadratic growth. The Appendix contains the proofs of the results of the paper.

\section{Notation and Definitions}

Let $E$ be a finite-dimensional real vector space with norm $\|\cdot\|$ and let $E^{*}$ be the conjugate space to $E$. Denote by $\langle s, x\rangle$ the value of linear function $s \in E^{*}$ at point $x \in E$ and by $\|\cdot\|_{*}$ the conjugate to 
norm $\|\cdot\|$ on $E^{*}$, i.e.,

$$
\|s\|_{*}=\max _{x}\{\langle s, x\rangle:\|x\| \leq 1\}, \quad s \in E^{*} .
$$

On the unit ball

$$
B=\{x \in E:\|x\| \leq 1\},
$$

we consider a continuous convex function $\theta: B \rightarrow \mathbf{R}$ with the following property:

$$
\left\langle\theta^{\prime}(x)-\theta^{\prime}\left(x^{\prime}\right), x-x^{\prime}\right\rangle \geq\left\|x-x^{\prime}\right\|^{2}, \quad \forall x, x^{\prime} \in B,
$$

where $\theta^{\prime}(\cdot)$ is a continuous in $B^{o}=\{x \in B: \partial \theta(x) \neq \emptyset\}$ version of the subgradient of $\theta(\cdot)$ and $\partial \theta(x)$ denotes the subdifferential of function $\theta(\cdot)$ at point $x$, i.e., the set of all subgradients at this point. In other words, function $\theta(\cdot)$ is strongly convex on $B$ with coefficient 1 with respect to the norm $\|\cdot\|$. We will call $\theta(\cdot)$ the normalized proxy function. Examples of such functions are:

- $\theta(x)=\frac{1}{2}\|x\|_{2}^{2}$ for $(E,\|\cdot\|)=\left(\mathbf{R}^{n},\|\cdot\|_{2}\right)$;

- $\theta(x)=2 \mathrm{e}(\ln \mathrm{n})\|x\|_{p}^{p}$ with $p=p(n):=1+\frac{1}{2 \ln n}$ for $(E,\|\cdot\|)=\left(\mathbf{R}^{n},\|\cdot\|_{1}\right)$;

- $\theta(x)=4 \mathrm{e}(\ln \mathrm{n}) \sum_{\mathrm{i}=1}^{\mathrm{n}}\left|\lambda_{\mathrm{i}}(\mathrm{x})\right|^{\mathrm{p}}$ with $p=p(n)$ for $E=S_{n}$, where $S_{n}$ is the space of symmetric $n \times n$ matrices equipped with the nuclear norm $\|x\|=\sum_{i=1}^{n}\left|\lambda_{i}(x)\right|$ and $\lambda_{i}(x)$ are eigenvalues of matrix $x$.

Here and in what follows, $\|\cdot\|_{p}$ denotes the $\ell_{p}$-norm in $\mathbf{R}^{n}, p \geq 1$. Without loss of generality, we will assume below that

$$
0=\arg \min _{x \in B} \theta(x) .
$$

We also introduce the notation

$$
\Theta=\max _{x \in B} \theta(x)-\min _{x \in B} \theta(x) \geq \frac{1}{2} .
$$

Now, let $X$ be a convex compact subset in $E$ and let $x_{0} \in X$ and $R>0$ be such that $\max _{x \in X}\left\|x-x_{0}\right\| \leq$ $R$. We equip $X$ with a proxy function

$$
\vartheta(x)=R^{2} \theta\left(\frac{x-x_{0}}{R}\right) .
$$

Note that $\vartheta(\cdot)$ is strongly convex with coefficient 1 and

$$
\max _{x \in X} \vartheta(x)-\min _{x \in X} \vartheta(x) \leq R^{2} \Theta .
$$

Let $D:=\max _{x, x^{\prime} \in X}\left\|x-x^{\prime}\right\|$ be the diameter of the set $X$. Then $D \leq 2 R$.

We will also use the Bregman divergence

$$
V_{x}(z)=\vartheta(z)-\vartheta(x)-\left\langle\vartheta^{\prime}(x), z-x\right\rangle, \quad \forall z, x \in X .
$$

In the following, we denote by $C$ and $C^{\prime}$ positive numerical constants, not necessarily the same in different cases. 


\section{Assumptions}

Consider a convex composite stochastic optimisation problem (1) on a convex compact set $X \subset E$. Assume in the following that the function

$$
\phi(x)=\mathbf{E}\{\Phi(x, \omega)\}
$$

is convex on $X$, differentiable at each point of the set $X$ and its gradient satisfies the Lipschitz condition

$$
\left\|\nabla \phi\left(x^{\prime}\right)-\nabla \phi(x)\right\|_{*} \leq L\left\|x-x^{\prime}\right\|, \quad \forall x, x^{\prime} \in X .
$$

Assume also that function $\psi$ is convex and continuous. In what follows, we assume that we have at our disposal a stochastic oracle, which for any input $(x, \omega) \in X \times \Omega$, returns a random vector $G(x, \omega)$, satisfying the conditions (2). In addition, it is assumed that for any $a \in E^{*}$ and $\beta>0$ an exact solution of the minimization problem

$$
\min _{z \in X}\{\langle a, z\rangle+\psi(z)+\beta \vartheta(z)\}
$$

is available. This assumption is fulfilled for typical penalty functions $\psi$, such as convex power functions of the $\ell_{p}$-norm (if $X$ is a convex compact in $\mathbf{R}^{n}$ ) or negative entropy $\psi(x)=\kappa \sum_{j=1}^{n} x_{j} \ln x_{j}$, where $\kappa>0$ (if $X$ is the standard simplex in $\mathbf{R}^{n}$ ). Finally, it is assumed that a vector $g(\bar{x})$ is available, where $\bar{x} \in X$ is a point in the set $X$ such that

$$
\|g(\bar{x})-\nabla \phi(\bar{x})\|_{*} \leq v \sigma
$$

with a constant $v \geq 0$. This assumption is motivated as follows.

First, if we a priori know that the global minimum of function $\phi$ is attained at an interior point $x_{\phi}$ of the set $X$ (what is common in statistical applications of stochastic approximation), we have $\nabla \phi\left(x_{\phi}\right)=0$. Therefore, choosing $\bar{x}=x_{\phi}$, one can put $g(\bar{x})=0$ and assumption (6) holds automatically with $v=0$.

Second, in general, one can choose $\bar{x}$ as any point of the set $X$ and $g(\bar{x})$ as a geometric median of stochastic gradients $G\left(\bar{x}, \omega_{i}\right), i=1, \ldots, m$, over $m$ oracle queries. It follows from [34] that if $m$ is of order $\ln \left(\varepsilon^{-1}\right)$ with some sufficiently small $\varepsilon>0$, then

$$
\operatorname{Prob}\left\{\|g(\bar{x})-\nabla \phi(\bar{x})\|_{*}>v \sigma\right\} \leq \varepsilon .
$$

Thus, the confidence bounds obtained below will remain valid up to an $\varepsilon$-correction in the probability of deviations.

\section{Accuracy bounds for Algorithm RSMD}

In what follows, we consider that the assumptions of Section 3 are fulfilled. Introduce a composite proximal transform

$$
\begin{aligned}
\operatorname{Prox}_{\beta, x}(\xi) & :=\arg \min _{z \in X}\left\{\langle\xi, z\rangle+\psi(z)+\beta V_{x}(z)\right\}= \\
& =\arg \min _{z \in X}\left\{\left\langle\xi-\beta \vartheta^{\prime}(x), z\right\rangle+\psi(z)+\beta \vartheta(z)\right\},
\end{aligned}
$$

where $\beta>0$ is a tuning parameter.

For $i=1,2, \ldots$, define the algorithm of Robust Stochastic Mirror Descent (RSMD) by the recursion

$$
x_{i}=\operatorname{Prox}_{\beta_{i-1}, x_{i-1}}\left(y_{i}\right), \quad x_{0} \in X,
$$




$$
y_{i}= \begin{cases}G\left(x_{i-1}, \omega_{i}\right), & \text { if }\left\|G\left(x_{i-1}, \omega_{i}\right)-g(\bar{x})\right\|_{*} \leq L\left\|\bar{x}-x_{i-1}\right\|+\lambda+v \sigma \\ g(\bar{x}), & \text { otherwise. }\end{cases}
$$

Here $\beta_{i}>0, i=0,1, \ldots$, and $\lambda>0$ are tuning parameters that will be defined below, and $\omega_{1}, \omega_{2}, \ldots$ are independent identically distributed (i.i.d.) realizations of a random variable $\omega$, corresponding to the oracle queries at each step of the algorithm.

The approximate solution of problem (1) after $N$ iterations is defined as the weighted average

$$
\widehat{x}_{N}=\left[\sum_{i=1}^{N} \beta_{i-1}^{-1}\right]^{-1} \sum_{i=1}^{N} \beta_{i-1}^{-1} x_{i} .
$$

If the global minimum of function $\phi$ is attained at an interior point of the set $X$ and $v=0$, then definition (10) is simplified. In this case, replacing $\left\|\bar{x}-x_{i-1}\right\|$ by the upper bound $D$ and putting $v=0$ and $g(\bar{x})=0$ in $(10)$, we define the truncated stochastic gradient by the formula

$$
y_{i}= \begin{cases}G\left(x_{i-1}, \omega_{i}\right), & \text { if }\left\|G\left(x_{i-1}, \omega_{i}\right)\right\|_{*} \leq L D+\lambda, \\ 0, & \text { otherwise. }\end{cases}
$$

The next result describes some useful properties of mirror descent recursion (9). Define

$$
\xi_{i}=y_{i}-\nabla \phi\left(x_{i-1}\right)
$$

and

$$
\varepsilon\left(x^{N}, z\right)=\sum_{i=1}^{N} \beta_{i-1}^{-1}\left[\left\langle\nabla \phi\left(x_{i-1}\right), x_{i}-z\right\rangle+\psi\left(x_{i}\right)-\psi(z)\right]+\frac{1}{2} V_{x_{i-1}}\left(x_{i}\right),
$$

where $x^{N}=\left(x_{0}, \ldots, x_{N}\right)$.

Proposition 1 Let $\beta_{i} \geq 2 L$ for all $i=0,1, \ldots$, and let $\widehat{x}_{N}$ be defined in (11), where $x_{i}$ are iterations (9) for any values $y_{i}$, not necessarily given by (10). Then for any $z \in X$ we have

$$
\begin{aligned}
{\left[\sum_{i=1}^{N} \beta_{i-1}^{-1}\right]\left[F\left(\widehat{x}_{N}\right)-F(z)\right] } & \leq \sum_{i=1}^{N} \beta_{i-1}^{-1}\left[F\left(x_{i}\right)-F(z)\right] \leq \varepsilon\left(x^{N}, z\right) \\
& \leq V_{x_{0}}(z)+\sum_{i=1}^{N}\left[\frac{\left\langle\xi_{i}, z-x_{i-1}\right\rangle}{\beta_{i-1}}+\frac{\left\|\xi_{i}\right\|_{*}^{2}}{\beta_{i-1}^{2}}\right] \\
& \leq 2 V_{x_{0}}(z)+\sum_{i=1}^{N}\left[\frac{\left\langle\xi_{i}, z_{i-1}-x_{i-1}\right\rangle}{\beta_{i-1}}+\frac{3}{2} \frac{\left\|\xi_{i}\right\|_{*}^{2}}{\beta_{i-1}^{2}}\right]
\end{aligned}
$$

where $z_{i}$ is a random vector with values in $X$ depending only on $x_{0}, \xi_{1}, \ldots, \xi_{i}$.

Using Proposition 1 we obtain the following bounds on the expected error $F\left(\widehat{x}_{N}\right)-F_{*}$ of the approximate solution of problem (1) based on the RSMD algorithm. In what follows, we denote by $\mathbf{E}\{\cdot\}$ the expectation with respect to the distribution of $\omega^{N}=\left(\omega_{1}, \ldots, \omega_{N}\right) \in \Omega^{\otimes N}$.

Corollary 1 Set $M=L R$. Assume that $\lambda \geq \max \{M, \sigma \sqrt{N}\}+v \sigma$ and $\beta_{i} \geq 2 L$ for all $i=0,1, \ldots$. Let $\widehat{x}_{N}$ be the approximate solution (11), where $x_{i}$ are the iterations of the RSMD algorithm defined by relations (9) and (10). Then

$$
\mathbf{E}\left\{F\left(\widehat{x}_{N}\right)\right\}-F_{*} \leq\left[\sum_{i=1}^{N} \beta_{i-1}^{-1}\right]^{-1}\left[R^{2} \Theta+\sum_{i=1}^{N}\left(\frac{2 D \sigma}{\beta_{i-1} \sqrt{N}}+\frac{4 \sigma^{2}}{\beta_{i-1}^{2}}\right)\right] .
$$


In particular, if $\beta_{i}=\bar{\beta}$ for all $i=0,1, \ldots$, where

$$
\bar{\beta}=\max \left\{2 L, \frac{\sigma \sqrt{N}}{R \sqrt{\Theta}}\right\},
$$

then the following inequalities hold:

$$
\mathbf{E}\left\{F\left(\widehat{x}_{N}\right)\right\}-F_{*} \leq \frac{\bar{\beta}}{N} \mathbf{E}\left\{\sup _{z \in X} \varepsilon\left(x^{N}, z\right)\right\} \leq C \max \left\{\frac{L R^{2} \Theta}{N}, \frac{\sigma R \sqrt{\Theta}}{\sqrt{N}}\right\} .
$$

Moreover, in this case we have the following inequality with explicit constants:

$$
\mathbf{E}\left\{F\left(\widehat{x}_{N}\right)\right\}-F_{*} \leq \max \left\{\frac{2 L R^{2} \Theta}{N}+\frac{4 R \sigma(1+\sqrt{\Theta})}{\sqrt{N}}, \frac{2 R \sigma(1+4 \sqrt{\Theta})}{\sqrt{N}}\right\} .
$$

This result shows that if the truncation threshold $\lambda$ is large enough, then the expected error of the proposed algorithm is bounded similarly to the expected error of the standard mirror descent algorithm with averaging, i.e., the algorithm in which stochastic gradients are taken without truncation: $y_{i}=$ $G\left(x_{i-1}, \omega_{i}\right)$.

The following theorem gives confidence bounds for the proposed algorithm.

Theorem 1 Let $\beta_{i}=\bar{\beta} \geq 2 L$ for all $i=0,1, \ldots$, and let $1 \leq \tau \leq N / v^{2}$,

$$
\lambda=\max \left\{\sigma \sqrt{\frac{N}{\tau}}, M\right\}+v \sigma .
$$

Let $\widehat{x}_{N}$ be the approximate solution (11), where $x_{i}$ are the RSMD iterations defined by relations (9) and (10). Then there is a random event $\mathcal{A}_{N} \subset \Omega^{\otimes N}$ of probability at least $1-2 e^{-\tau}$ such that for all $\omega^{N} \in \mathcal{A}_{N}$ the following inequalities hold:

$$
\begin{aligned}
F\left(\widehat{x}_{N}\right)-F_{*} & \leq \frac{\bar{\beta}}{N} \sup _{z \in X} \varepsilon\left(x^{N}, z\right) \leq \\
& \leq \frac{C}{N}\left(\bar{\beta} R^{2} \Theta+R \max \{\sigma \sqrt{\tau N}, M \tau\}+\bar{\beta}^{-1} \max \left\{N \sigma^{2}, M^{2} \tau\right\}\right) .
\end{aligned}
$$

In paticular, chosing $\bar{\beta}$ as in formula (16) we have, for all $\omega^{N} \in \mathcal{A}_{N}$,

$$
F\left(\widehat{x}_{N}\right)-F_{*} \leq \max \left\{C_{1} \frac{L R^{2}[\tau \vee \Theta]}{N}, C_{2} \sigma R \sqrt{\frac{\tau \vee \Theta}{N}}\right\},
$$

where $C_{1}>0$ and $C_{2}>0$ are numerical constants.

The values of the numerical constants $C_{1}$ and $C_{2}$ in (19) can be obtained from the proof of the theorem, cf. the bound in (40).

Confidence bound (19) in Theorem 1 contains two terms corresponding to the deterministic error and to the stochastic error. Unlike the case of noise with a "light tail" (see, for example, [40]) and the bound in expectation (17), the deterministic error $L R^{2}[\tau \vee \Theta] / N$ depends on $\tau$. Note also that Theorem 1 gives a sub-Gaussian confidence bound (the order of the stochastic error is $\sigma R \sqrt{[\tau \vee \Theta] / N}$ ). However, the truncation threshold $\lambda$ depends on the confidence level $\tau$. This can be inconvenient for the implementation of the algorithms. Some simple but coarser confidence bounds can be obtained by using a universal threshold independent of $\tau$, which is $\lambda=\max \{\sigma \sqrt{N}, M\}+v \sigma$. In particular, we have the following result. 
Theorem 2 Let $\beta_{i}=\bar{\beta} \geq 2 L$ for all $i=0,1, \ldots$, and let $N \geq v^{2}$. Set

$$
\lambda=\max \{\sigma \sqrt{N}, M\}+v \sigma .
$$

Let $\widehat{x}_{N}=N^{-1} \sum_{i=1}^{N} x_{i}$, where $x_{i}$ are the iterations of the RSMD algorithm defined by relations (9) and (10). Then there is a random event $\mathcal{A}_{N} \subset \Omega^{\otimes N}$ of probability at least $1-2 e^{-\tau}$ such that for all $\omega^{N} \in \mathcal{A}_{N}$ the following inequalities hold:

$$
\begin{aligned}
F\left(\widehat{x}_{N}\right)-F_{*} & \leq \frac{\bar{\beta}}{N} \sup _{z \in X} \varepsilon\left(x^{N}, z\right) \leq \\
& \leq \frac{C}{N}\left(\bar{\beta} R^{2} \Theta+\tau R \max \{\sigma \sqrt{N}, M\}+\tau \bar{\beta}^{-1} \max \left\{N \sigma^{2}, M^{2}\right\}\right) .
\end{aligned}
$$

In particular, choosing $\bar{\beta}$ as in formula (16) we have

$$
F\left(\widehat{x}_{N}\right)-F_{*} \leq \frac{\bar{\beta}}{N} \sup _{z \in X} \varepsilon\left(x^{N}, z\right) \leq C \max \left\{\frac{L R^{2}[\tau \vee \Theta]}{N}, \tau \sigma R \sqrt{\frac{\Theta}{N}}\right\}
$$

for all $\omega^{N} \in \mathcal{A}_{N}$.

The values of the numerical constants $C$ in Theorem 2 can be obtained from the proof, cf. the bound in (40).

\section{Robust Confidence Bounds for Stochastic Optimization Methods}

Consider an arbitrary algorithm for solving the problem (1) based on $N$ queries of the stochastic oracle. Assume that we have a sequence $\left(x_{i}, G\left(x_{i}, \omega_{i+1}\right)\right), i=0, \ldots, N$, where $x_{i} \in X$ are the search points of some stochastic algorithm and $G\left(x_{i}, \omega_{i+1}\right)$ are the corresponding observations of the stochastic gradient. It is assumed that $x_{i}$ depends only on $\left\{\left(x_{j-1}, \omega_{j}\right), j=1, \ldots, i\right\}$. The approximate solution of the problem (1) is defined in the form:

$$
\widehat{x}_{N}=N^{-1} \sum_{i=1}^{N} x_{i}
$$

Our goal is to construct a confidence interval with sub-Gaussian accuracy for $F\left(\widehat{x}_{N}\right)-F_{*}$. To do this, we use the following fact. Note that for any $t \geq L$ the value

$$
\epsilon_{N}(t)=N^{-1} \sup _{z \in X}\left\{\sum_{i=1}^{N}\left[\left\langle\nabla \phi\left(x_{i-1}\right), x_{i}-z\right\rangle+\psi\left(x_{i}\right)-\psi(z)+t V_{x_{i-1}}\left(x_{i}\right)\right]\right\}
$$

is an upper bound on the accuracy of the approximate solution $\widehat{x}_{N}$ :

$$
F\left(\widehat{x}_{N}\right)-F_{*} \leq \epsilon_{N}(t)
$$

(see Lemma 1 in Appendix). This fact is true for any sequence of points $x_{0}, \ldots, x_{N}$ in $X$, regardless of how they are obtained. However, since the function $\nabla \phi(\cdot)$ is not known, the estimate (22) cannot be used in practice. Replacing the gradients $\nabla \phi\left(x_{i-1}\right)$ in $(21)$ with their truncated estimates $y_{i}$ defined in (10) we get an implementable analogue of $\epsilon_{N}(t)$ :

$$
\widehat{\epsilon}_{N}(t)=N^{-1} \sup _{z \in X}\left\{\sum_{i=1}^{N}\left[\left\langle y_{i}, x_{i}-z\right\rangle+\psi\left(x_{i}\right)-\psi(z)+t V_{x_{i-1}}\left(x_{i}\right)\right]\right\} .
$$


Note that computing $\widehat{\epsilon}_{N}(t)$ reduces to solving a problem of the form (8) with $\beta=0$. Thus, it is computationally not more complex than, for example, one step of the RSMD algorithm. Replacing $\nabla \phi\left(x_{i-1}\right)$ with $y_{i}$ introduces a random error. In order to get a reliable upper bound for $\epsilon_{N}(t)$, we need to compensate this error by slightly increasing $\widehat{\epsilon}_{N}(t)$. Specifically, we add to $\widehat{\epsilon}_{N}(t)$ the value

$$
\begin{aligned}
\bar{\rho}_{N}(\tau)= & 4 R \sqrt{5 \Theta \max \left\{N \sigma^{2}, M^{2} \tau\right\}}+16 R \max \{\sigma \sqrt{N \tau}, M \tau\}+ \\
& +\min _{\mu \geq 0}\left\{20 \mu \max \left\{N \sigma^{2}, M^{2} \tau\right\}+\mu^{-1} \sum_{i=1}^{N} V_{x_{i-1}}\left(x_{i}\right)\right\},
\end{aligned}
$$

where $\tau>0$.

Proposition 2 Let $\left(x_{i}, G\left(x_{i}, \omega_{i+1}\right)\right)_{i=0}^{N}$ be the trajectory of a stochastic algorithm for which $x_{i}$ depends only on $\left\{\left(x_{j-1}, \omega_{j}\right), j=1, \ldots, i\right\}$. Let $0<\tau \leq N / v^{2}$ and let $y_{i}=y_{i}(\tau)$ be truncated stochastic gradients defined in (10), where the threshold $\lambda=\lambda(\tau)$ is chosen in the form (18). Then for any $t \geq L$ the value

$$
\Delta_{N}(\tau, t)=\widehat{\epsilon}_{N}(t)+\bar{\rho}_{N}(\tau) / N
$$

is an upper bound for $\epsilon_{N}(t)$ with probability $1-2 \mathrm{e}^{-\tau}$, so that

$$
\operatorname{Prob}\left\{F\left(\widehat{x}_{N}\right)-F_{*} \leq \Delta_{N}(\tau, t)\right\} \geq 1-2 \mathrm{e}^{-\tau} .
$$

Since $\Delta_{N}(\tau, t)$ monotonically increases in $t$ it suffices to use this bound for $t=L$ when $L$ is known. Note that, although $\Delta_{N}(\tau, t)$ gives an upper bound for $\epsilon_{N}(t)$, Proposition 2 does not guarantee that $\Delta_{N}(\tau, t)$ is sufficiently close to $\epsilon_{N}(t)$. However, this property holds for the RSMD algorithm with a constant step, as follows from the next result.

Corollary 2 Under the conditions of Proposition 2, let the vectors $x_{0}, \ldots, x_{N}$ be given by the RSMD recursion (9)-(10), where $\beta_{i}=\bar{\beta} \geq 2 L, i=0, \ldots, N-1$. Then

$$
\begin{aligned}
\bar{\rho}_{N}(\tau) \leq & N \epsilon_{N}(\bar{\beta})+4 R \sqrt{5 \Theta \max \left\{N \sigma^{2}, M^{2} \tau\right\}}+ \\
& +16 R \max \{\sigma \sqrt{N \tau}, M \tau\}+20 \bar{\beta}^{-1} \max \left\{N \sigma^{2}, M^{2} \tau\right\} .
\end{aligned}
$$

Moreover, if $\bar{\beta} \geq \max \left\{2 L, \frac{\sigma \sqrt{N}}{R \sqrt{\Theta}}\right\}$ then

$$
\bar{\rho}_{N}(\tau) \leq N \epsilon_{N}(\bar{\beta})+C_{3} L R^{2}[\Theta \vee \tau]+C_{4} \sigma R \sqrt{N[\Theta \vee \tau]},
$$

and with probability at least $1-4 \mathrm{e}^{-\tau}$ the value $\Delta_{N}(\tau, \bar{\beta})$ satisfies the inequalities

$$
\epsilon_{N}(\bar{\beta}) \leq \Delta_{N}(\tau, \bar{\beta}) \leq 3 \epsilon_{N}(\bar{\beta})+2 C_{3} \frac{L R^{2}[\Theta \vee \tau]}{N}+2 C_{4} \sigma R \sqrt{\frac{[\Theta \vee \tau]}{N}},
$$

where $C_{3}>0$ and $C_{4}>0$ are numerical constants.

The values of the numerical constants $C_{3}$ and $C_{4}$ can be derived from the proof of this corollary. 


\section{Robust Confidence Bounds for Quadratic Growth Problems}

In this section, it is assumed that $F$ is a function with quadratic growth on $X$ in the following sense (cf. [41]). Let $F$ be a continuous function on $X$ and let $X_{*} \subset X$ be the set of its minimizers on $X$. Then $F$ is called a function with quadratic growth on $X$ if there is a constant $\kappa>0$ such that for any $x \in X$ there exists $\bar{x}(x) \in X_{*}$ such that the following inequality holds:

$$
F(x)-F_{*} \geq \frac{\kappa}{2}\|x-\bar{x}(x)\|^{2} .
$$

Note that every strongly convex function $F$ on $X$ with the strong convexity coefficient $\kappa$ is a function with quadratic growth on $X$. However, the assumption of strong convexity, when used together with the Lipschitz condition with constant $L$ on the gradient of $F$, has the disadvantage that, except for the case when $\|\cdot\|$ is the Euclidean norm, the ratio $L / \kappa$ depends on the dimension of the space $E$. For example, in the important cases where $\|\cdot\|$ is the $\ell_{1}$-norm, the nuclear norm, the total variation norm, etc., one can easily check (cf. [2]) that there are no functions with Lipschitz continuous gradient such that the ratio $L / \kappa$ is smaller than the dimension of the space. Replacing the strong convexity with the growth condition (26) eliminates this problem, see the examples in [41]. On the other hand, assumption (26) is quite natural in the composite optimization problem since in many interesting examples the function $\phi$ is smooth and the non-smooth part $\psi$ of the objective function is strongly convex. In particular, if $E=\mathbf{R}^{n}$ and the norm is the $\ell_{1}$-norm, this allows us to consider such strongly convex components as the negative entropy $\psi(x)=\kappa \sum_{j} x_{j} \ln x_{j}$ (if $X$ is standard simplex in $\mathbf{R}^{n}$ ), $\psi(x)=\gamma(\kappa)\|x\|_{p}^{p}$ with $1 \leq p \leq 2$ and with the corresponding choice of $\gamma(\kappa)>0$ (if $X$ is a convex compact in $\mathbf{R}^{n}$ ) and others. In all these cases, condition (26) is fulfilled with a known constant $\kappa$, which allows for the use of the approach of $[2,42]$ to improve the confidence bounds of the stochastic mirror descent.

The RSMD algorithm for quadratically growing functions will be defined in stages. At each stage, for specially selected $r>0$ and $y \in X$ it solves an auxiliary problem

$$
\min _{x \in X_{r}(y)} F(x)
$$

using the RSMD. Here

$$
X_{r}(y)=\{x \in X:\|x-y\| \leq r\} .
$$

We initialize the algorithm by choosing arbitrary $y_{0}=x_{0} \in X$ and $r_{0} \geq \max _{z \in X}\left\|z-x_{0}\right\|$. We set $r_{k}^{2}=2^{-k} r_{0}^{2}, k=1,2, \ldots$ Let $C_{1}$ and $C_{2}$ be the numerical constants in the bound (19) of Theorem 1 . For a given parameter $\tau>0$, and $k=1,2, \ldots$ we define the values

$$
\left.\bar{N}_{k}=\max \left\{4 C_{1} \frac{L[\tau \vee \Theta]}{\kappa}, 16 C_{2} \frac{\sigma^{2}[\tau \vee \Theta]}{\kappa^{2} r_{k-1}^{2}}\right\}, \quad N_{k}=\right\rfloor \bar{N}_{k}\lfloor.
$$

Here $\rfloor t\lfloor$ denotes the smallest integer greater than or equal to $t$. Set

$$
m(N):=\max \left\{k: \sum_{j=1}^{k} N_{j} \leq N\right\} .
$$

Now, let $k \in\{1,2, \ldots, m(N)\}$. At the $k$-th stage of the algorithm, we solve the problem of minimization of $F$ on the ball $X_{r_{k-1}}\left(y_{k-1}\right)$, we find its approximate solution $\widehat{x}_{N_{k}}$ according to (9)-(11), where we replace $x_{0}$ by $y_{k-1}, X$ by $X_{r_{k-1}}\left(y_{k-1}\right), R$ by $r_{k-1}, N$ by $N_{k}$, and set

$$
\lambda=\max \left\{\sigma \sqrt{\frac{N}{\tau}}, L r_{k-1}\right\}+v \sigma,
$$


and

$$
\beta_{i} \equiv \max \left\{2 L, \frac{\sigma \sqrt{N}}{r_{k-1} \sqrt{\Theta}}\right\} .
$$

It is assumed that, at each stage $k$ of the algorithm, an exact solution of the minimization problem

$$
\min _{z \in X_{r_{k-1}}\left(y_{k-1}\right)}\{\langle a, z\rangle+\psi(z)+\beta \vartheta(z)\}
$$

is available for any $a \in E$ and $\beta>0$. At the output of the $k$-th stage of the algorithm, we obtain $y_{k}:=\widehat{x}_{N_{k}}$.

Theorem 3 Assume that $m(N) \geq 1$, i.e. at least one stage of the algorithm described above is completed. Then there is a random event $\mathcal{B}_{N} \subset \Omega^{\otimes N}$ of probability at least $1-2 m(N) e^{-\tau}$ such that for $\omega^{N} \in \mathcal{B}_{N}$ the approximate solution $y_{m(N)}$ after $m(N)$ stages of the algorithm satisfies the inequality

$$
F\left(y_{m(N)}\right)-F_{*} \leq C \max \left\{\kappa r_{0}^{2} 2^{-N / 4}, \kappa r_{0}^{2} \exp \left(-\frac{C^{\prime} \kappa N}{L[\tau \vee \Theta]}\right), \frac{\sigma^{2}[\tau \vee \Theta]}{\kappa N}\right\} .
$$

Theorem 3 shows that, for functions with quadratic growth, the deterministic error component can be significantly reduced - it becomes exponentially decreasing in $N$. The stochastic error component is also significantly reduced. Note that the factor $m(N)$ is of logarithmic order and has little effect on the probability of deviations. Indeed, it follows from $(27)$ that $m(N) \leq C \ln \left(\frac{C^{\prime} \kappa^{2} r_{0}^{2} N}{\sigma^{2}(\tau \vee \Theta)}\right)$. Neglecting this factor in the probability of deviations and considering the stochastic component of the error, we see that the confidence bound of Theorem 3 is approximately sub-exponential rather than sub-Gaussian.

\section{Conclusion}

We have considered algorithms of smooth stochastic optimization when the distribution of noise in observations has heavy tails. It is shown that by truncating the observed gradients with a suitable threshold one can construct confidence sets for the approximate solutions that are similar to those in the case of "light tails". It should be noted that the order of the deterministic error in the obtained bounds is suboptimal - it is substantially greater than the optimal rates achieved by the accelerated algorithms [3, 40], namely, $O\left(L R^{2} N^{-2}\right)$ in the case of convex objective function and $O(\exp (-N \sqrt{\kappa / L}))$ in the strongly convex case. On the other hand, the proposed approach cannot be used to obtain robust versions of the accelerated algorithms since applying it to such algorithms leads to accumulation of the bias caused by the truncation of the gradients. The problem of constructing accelerated robust stochastic algorithms with optimal guarantees remains open.

APPENDIX

A.1. Preliminary remarks. We start with the following known result.

Lemma 1 Assume that $\phi$ and $\psi$ satisfy the assumptions of Section 3, and let $x_{0}, \ldots, x_{N}$ be some points of the set $X$. Define

$$
\varepsilon_{i+1}(z):=\left\langle\nabla \phi\left(x_{i}\right), x_{i+1}-z\right\rangle+\left\langle\psi^{\prime}\left(x_{i+1}\right), x_{i+1}-z\right\rangle+L V_{x_{i}}\left(x_{i+1}\right) .
$$

Then for any $z \in X$ the following inequality holds:

$$
F\left(x_{i+1}\right)-F(z) \leq \varepsilon_{i+1}(z) .
$$

In addition, for $\widehat{x}_{N}=\frac{1}{N} \sum_{i=1}^{N} x_{i}$ we have

$$
F\left(\widehat{x}_{N}\right)-F(z) \leq N^{-1} \sum_{i=1}^{N}\left[F\left(x_{i}\right)-F(z)\right] \leq N^{-1} \sum_{i=0}^{N-1} \varepsilon_{i+1}(z) .
$$


Proof Using the property $V_{x}(z) \geq \frac{1}{2}\|x-z\|^{2}$, the convexity of functions $\phi$ and $\psi$ and the Lipschitz condition on $\nabla \phi$ we get that, for any $z \in X$,

$$
\begin{aligned}
F\left(x_{i+1}\right)-F(z) & =\left[\phi\left(x_{i+1}\right)-\phi(z)\right]+\left[\psi\left(x_{i+1}\right)-\psi(z)\right]= \\
& =\left[\phi\left(x_{i+1}\right)-\phi\left(x_{i}\right)\right]+\left[\phi\left(x_{i}\right)-\phi(z)\right]+\left[\psi\left(x_{i+1}\right)-\psi(z)\right] \leq \\
& \leq\left[\left\langle\nabla \phi\left(x_{i}\right), x_{i+1}-x_{i}\right\rangle+L V_{x_{i}}\left(x_{i+1}\right)\right]+\left\langle\nabla \phi\left(x_{i}\right), x_{i}-z\right\rangle+\psi\left(x_{i+1}\right)-\psi(z) \leq \\
& \leq\left\langle\nabla \phi\left(x_{i}\right), x_{i+1}-z\right\rangle+\left\langle\psi^{\prime}\left(x_{i+1}\right), x_{i+1}-z\right\rangle+L V_{x_{i}}\left(x_{i+1}\right)=\varepsilon_{i+1}(z) .
\end{aligned}
$$

Summing up over $i$ from 0 to $N-1$ and using the convexity of $F$ we obtain the second result of the lemma.

In what follows, we denote by $\mathbf{E}_{x_{i}}\{\cdot\}$ the conditional expectation for fixed $x_{i}$.

Lemma 2 Let the assumptions of Section 3 be fulfilled and let $x_{i}$ and $y_{i}$ satisfy the RSMD recursion, cf. (9) and (10). Then

(a) $\quad\left\|\xi_{i}\right\|_{*} \leq 2(M+v \sigma)+\lambda$,

(b) $\quad\left\|\mathbf{E}_{x_{i-1}}\left\{\xi_{i}\right\}\right\|_{*} \leq(M+v \sigma)\left(\frac{\sigma}{\lambda}\right)^{2}+\frac{\sigma^{2}}{\lambda}$,

(c) $\quad\left(\mathbf{E}_{x_{i-1}}\left\{\left\|\xi_{i}\right\|_{*}^{2}\right\}\right)^{1 / 2} \leq \sigma+(M+v \sigma) \frac{\sigma}{\lambda}$.

Proof Set $\chi_{i}=1_{\left\|G\left(x_{i-1}, \omega_{i}\right)-g(\bar{x})\right\|_{*}>L\left\|x_{i-1}-\bar{x}\right\|+\lambda+v \sigma}$. Note that by construction $\chi_{i} \leq \eta_{i}:=1_{\| G\left(x_{i-1}, \omega_{i}\right)-\nabla f\left(x_{i-1} \|_{*}>\lambda\right.}$. We have

$$
\begin{aligned}
\xi_{i} & =y_{i}-\nabla \phi\left(x_{i-1}\right)=\left[G\left(x_{i-1}, \omega_{i}\right)-\nabla \phi\left(x_{i-1}\right)\right]\left(1-\chi_{i}\right)+\left[g(\bar{x})-\nabla \phi\left(x_{i-1}\right)\right] \chi_{i}= \\
& =\left[G\left(x_{i-1}, \omega_{i}\right)-g(\bar{x})\right]\left(1-\chi_{i}\right)+\left[g(\bar{x})-\nabla \phi\left(x_{i-1}\right)\right]= \\
& =\left[G\left(x_{i-1}, \omega_{i}\right)-\nabla \phi\left(x_{i-1}\right)\right]+\left[g(\bar{x})-G\left(x_{i-1}, \omega_{i}\right)\right] \chi_{i} .
\end{aligned}
$$

Therefore,

$$
\left\|\xi_{i}\right\|_{*} \leq\left\|\left[G\left(x_{i-1}, \omega_{i}\right)-g(\bar{x})\right]\left(1-\chi_{i}\right)\right\|_{*}+\left\|g(\bar{x})-\nabla \phi\left(x_{i-1}\right)\right\|_{*} \leq 2(M+v \sigma)+\lambda .
$$

Moreover, since $\mathbf{E}_{x_{i-1}}\left\{G\left(x_{i-1}, \omega_{i}\right)\right\}=\nabla \phi\left(x_{i-1}\right)$ we have

$$
\begin{aligned}
\left\|\mathbf{E}_{x_{i-1}}\left\{\xi_{i}\right\}\right\|_{*} & =\left\|\mathbf{E}_{x_{i-1}}\left\{\left[\left(G\left(x_{i-1}, \omega_{i}\right)-\nabla \phi\left(x_{i-1}\right)\right)-\left(g(\bar{x})-\nabla \phi\left(x_{i-1}\right)\right)\right] \chi_{i}\right\}\right\|_{*} \leq \\
& \leq \mathbf{E}_{x_{i-1}}\left\{\left[\left\|G\left(x_{i-1}, \omega_{i}\right)-\nabla \phi\left(x_{i-1}\right)\right\|_{*}+\left\|g(\bar{x})-\nabla \phi\left(x_{i-1}\right)\right\|_{*}\right] \chi_{i}\right\} \leq \\
& \leq \mathbf{E}_{x_{i-1}}\left\{\left\|G\left(x_{i-1}, \omega_{i}\right)-\nabla \phi\left(x_{i-1}\right)\right\|_{*} \eta_{i}\right\}+(M+v \sigma) \mathbf{E}_{x_{i-1}}\left\{\eta_{i}\right\} \leq \\
& \leq \frac{\sigma^{2}}{\lambda}+(M+v \sigma)\left(\frac{\sigma}{\lambda}\right)^{2} .
\end{aligned}
$$

Further,

$$
\left\|\xi_{i}\right\|_{*} \leq\left\|G\left(x_{i-1}, \omega_{i}\right)-\nabla \phi\left(x_{i-1}\right)\right\|_{*}(1-\chi)+\left\|g(\bar{x})-\nabla \phi\left(x_{i-1}\right)\right\|_{*} \chi_{i},
$$

and

$$
\begin{aligned}
\mathbf{E}_{x_{i-1}}\left\{\left\|\xi_{i}\right\|_{*}^{2}\right\}^{1 / 2} \leq & \mathbf{E}_{x_{i-1}}\left\{\left\|G\left(x_{i-1}, \omega_{i}\right)-\nabla \phi\left(x_{i-1}\right)\right\|_{*}^{2}\right\}^{1 / 2}+ \\
& \quad+\mathbf{E}_{x_{i-1}}\left\{\left\|g(\bar{x})-\nabla \phi\left(x_{i-1}\right)\right\|_{*}^{2} \chi_{i}\right\}^{1 / 2} \leq \\
\leq & \sigma+(M+v \sigma) \mathbf{E}\left\{\chi_{i}\right\}^{1 / 2} \leq \sigma+(M+v \sigma) \mathbf{E}_{x_{i-1}}\left\{\eta_{i}\right\}^{1 / 2} \leq \\
\leq & \sigma+(M+v \sigma) \frac{\sigma}{\lambda} .
\end{aligned}
$$


The following lemma gives bounds for the deviations of the sums $\sum_{i}\left\langle\xi_{i}, x_{i-1}-z\right\rangle$ and $\sum_{i}\left\|\xi_{i}\right\|_{*}^{2}$.

Lemma 3 Let the assumptions of Section 3 be fulfilled and let $x_{i}$ and $y_{i}$ satisfy the recursion of RSMD, cf. (9) and (10).

(i) If $\tau \leq N / v^{2}$ and $\lambda=\max \left\{\sigma \sqrt{\frac{N}{\tau}}, M\right\}+v \sigma$ then, for any $z \in X$,

$$
\operatorname{Prob}\left\{\sum_{i=1}^{N}\left\langle\xi_{i}, z-x_{i-1}\right\rangle \geq 16 R \max \{\sigma \sqrt{N \tau}, M \tau\}\right\} \leq e^{-\tau},
$$

and

$$
\operatorname{Prob}\left\{\sum_{i=1}^{N}\left\|\xi_{i}\right\|_{*}^{2} \geq 40 \max \left\{N \sigma^{2}, M^{2} \tau\right\}\right\} \leq e^{-\tau} .
$$

(ii) If $N \geq v^{2}$ and $\lambda=\max \{\sigma \sqrt{N}, M\}+v \sigma$ then, for any $z \in X$,

$$
\operatorname{Prob}\left\{\sum_{i=1}^{N}\left\langle\xi_{i}, z-x_{i-1}\right\rangle \geq 8(1+\tau) R \max \{\sigma \sqrt{N}, M\}\right\} \leq e^{-\tau}
$$

and

$$
\operatorname{Prob}\left\{\sum_{i=1}^{N}\left\|\xi_{i}\right\|_{*}^{2} \geq 8(2+3 \tau) \max \left\{N \sigma^{2}, M^{2}\right\}\right\} \leq e^{-\tau} .
$$

Proof Set $\zeta_{i}=\left\langle\xi_{i}, z-x_{i-1}\right\rangle$ and $\varsigma_{i}=\left\|\xi_{i}\right\|_{*}^{2}, i=1,2, \ldots$ Using Lemma 2 it is easy to check that the following inequalities are fulfilled

$$
\begin{aligned}
\text { (a) } & \left|\mathbf{E}_{x_{i-1}}\left\{\zeta_{i}\right\}\right| & \leq D\left[(M+v \sigma)(\sigma / \lambda)^{2}+\sigma^{2} / \lambda\right], \\
(b) & \left|\zeta_{i}\right| & \leq D[2(M+v \sigma)+\lambda], \\
(c) & \left(\mathbf{E}_{x_{i-1}}\left\{\zeta_{i}^{2}\right\}\right)^{1 / 2} & \leq D[\sigma+(M+v \sigma) \sigma / \lambda]
\end{aligned}
$$

and

$$
\begin{aligned}
(a) & \mathbf{E}_{x_{i-1}}\left\{\varsigma_{i}\right\} & \leq[\sigma+(M+v \sigma) \sigma / \lambda]^{2}, \\
(b) & \varsigma_{i} & \leq[2(M+v \sigma)+\lambda]^{2}, \\
(c) & \left(\mathbf{E}_{x_{i-1}}\left\{\varsigma_{i}^{2}\right\}\right)^{1 / 2} & \leq[\sigma+(M+v \sigma) \sigma / \lambda][2(M+v \sigma)+\lambda] .
\end{aligned}
$$

In what follows, we apply several times the Bernstein inequality, and each time we will use the same notation $r, A, s$ for the values that are, respectively, the uniform upper bound of the expectation, the maximum absolute value, and the standard deviation of a random variable.

$\mathbf{1}^{o}$. We first prove the statement $(i)$. We start with the case $M \leq \sigma \sqrt{\frac{N}{\tau}}$. It follows from (34) that in this case

$$
\begin{aligned}
\left|\mathbf{E}_{x_{i-1}}\left\{\zeta_{i}\right\}\right| & \leq 2 D \sigma^{2} / \lambda \leq 4 R \sigma \sqrt{\frac{\tau}{N}}=: r, \\
\left|\zeta_{i}\right| & \leq A:=3 \lambda D \leq 6 R \lambda, \\
\left(\mathbf{E}_{x_{i-1}}\left\{\zeta_{i}^{2}\right\}\right)^{1 / 2} & \leq s:=2 D \sigma \leq 4 R \sigma .
\end{aligned}
$$


Using (36) and Bernstein's inequality for martingales (see, for example, [43]) we get

$$
\begin{aligned}
\operatorname{Prob}\left\{\sum_{i=1}^{N} \zeta_{i} \geq 16 R \sigma \sqrt{N \tau}\right\} & \leq \operatorname{Prob}\left\{\sum_{i=1}^{N} \zeta_{i} \geq N r+3 s \sqrt{N \tau}\right\} \leq \\
& \leq \exp \left\{-\frac{9 \tau}{\left.2+\frac{2}{3} \frac{3 \sqrt{\tau} A}{s \sqrt{N}}\right\} \leq}\right. \\
& \leq \exp \left\{-\frac{9 \tau}{2+3(1+v \sqrt{\tau / N})}\right\} \leq e^{-\tau}
\end{aligned}
$$

for all $\tau>0$ satisfying the condition $\tau \leq 16 N /\left(9 v^{2}\right)$. On the other hand, in the case under consideration, the following inequalities hold (cf. (35) and (36))

$$
\mathbf{E}_{x_{i-1}}\left\{\varsigma_{i}\right\} \leq \underbrace{4 \sigma^{2}}_{=: r}, \quad \varsigma_{i} \leq \underbrace{9 \lambda^{2}}_{=: A}, \quad\left(\mathbf{E}_{x_{i-1}}\left\{\varsigma_{i}^{2}\right\}\right)^{1 / 2} \leq \underbrace{6 \lambda \sigma}_{=: s} .
$$

Thus,

$$
N r+3 s \sqrt{\tau N}=4 N \sigma^{2}+18 \lambda \sigma \sqrt{\tau N}=22 N \sigma^{2}+18 v \sigma^{2} \sqrt{N \tau} \leq 40 N \sigma^{2}
$$

for $0<\tau \leq N / v^{2}$. Applying again the Bernstein inequality, we get

$$
\operatorname{Prob}\left\{\sum_{i=1}^{N} \varsigma_{i} \geq 40 N \sigma^{2}\right\} \leq \exp \left\{-\frac{9 \tau}{2+(3+3 v \sqrt{\tau / N})}\right\} \leq e^{-\tau}
$$

for all $\tau>0$ satisfying the condition $\tau \leq N / v^{2}$.

$\mathbf{2}^{o}$. Assume now that $M>\sigma \sqrt{\frac{N}{\tau}}$, so that $\lambda=M+v \sigma$ and $\sigma^{2} \leq M^{2} \tau / N$. Then

$$
\begin{gathered}
\left|\mathbf{E}_{x_{i-1}} \zeta_{i}\right| \leq 4 R \sigma^{2} / \lambda \leq \underbrace{4 R M \tau / N}_{=: r},\left|\zeta_{i}\right| \leq R(2(M+v \sigma)+\lambda)=\underbrace{6 R(M+v \sigma)}_{=: A}, \\
\left(\mathbf{E}_{x_{i-1}}\left\{\zeta_{i}^{2}\right\}\right)^{1 / 2} \leq 4 R \sigma \leq \underbrace{4 M R \sqrt{\tau / N}}_{=: s} .
\end{gathered}
$$

Further,

$$
N r+3 s \sqrt{\tau N}=4 R M \tau+12 R M \tau=16 R M \tau,
$$

and applying again the Bernstein inequality we get

$$
\begin{aligned}
\operatorname{Prob}\left\{\sum_{i=1}^{N} \zeta_{i} \geq 16 R M \tau\right\} & \leq \exp \left\{-\frac{9 \tau}{2+\frac{2}{3} \frac{3 \sqrt{\tau} A}{s \sqrt{N}}}\right\} \leq \exp \left\{-\frac{9 \tau}{2+(3+3 v \sigma / M)}\right\} \leq \\
& \leq \exp \left\{-\frac{9 \tau}{5+3 v \sqrt{\tau / N}}\right\} \leq e^{-\tau}
\end{aligned}
$$

for all $\tau>0$, satisfying the condition $\tau \leq 16 N /\left(9 v^{2}\right)$. Next, in this case

$$
\mathbf{E}_{x_{i-1}}\left\{\varsigma_{i}\right\} \leq 4 \sigma^{2} \leq \underbrace{4 \tau M^{2} / N}_{=: r}, \quad \varsigma_{i} \leq \underbrace{9 \lambda^{2}}_{=: A}, \quad\left(\mathbf{E}_{x_{i-1}}\left\{\varsigma_{i}^{2}\right\}\right)^{1 / 2} \leq \underbrace{6 \lambda \sigma}_{=: s} \leq 6 \lambda M \sqrt{\tau / N} .
$$


Now,

$$
N r+3 s \sqrt{\tau N}=4 \tau M^{2}+18 \lambda \sigma \sqrt{\tau N} \leq 22 M^{2} \tau+18 v \sigma^{2} \sqrt{N \tau} \leq 40 M^{2} \tau,
$$

for $\tau \leq N / v^{2}$. Applying once again the Bernstein inequality we get

$$
\operatorname{Prob}\left\{\sum_{i=1}^{N} \varsigma_{i} \geq 40 \tau M^{2}\right\} \leq \exp \left\{-\frac{9 \tau}{2+(3+3 v \sqrt{\tau / N})}\right\} \leq e^{-\tau},
$$

for all $\tau>0$ satisfying the condition $\tau \leq N / v^{2}$.

$\mathbf{3}^{o}$. Now, consider the case $\lambda=\max \{M, \sigma \sqrt{N}\}+\sigma v$. Let $M \leq \sigma \sqrt{N}$, so that $\lambda=\sigma(\sqrt{N}+v)$. We argue in the same way as in the proof of $(i)$. By virtue of (34) we have

$$
\begin{aligned}
\left|\mathbf{E}_{x_{i-1}}\left\{\zeta_{i}\right\}\right| & \leq 4 R \frac{\sigma}{\sqrt{N}}=: r \\
\left(\mathbf{E}_{x_{i-1}}\left\{\zeta_{i}^{2}\right\}\right)^{1 / 2} & \leq 4 R \sigma=: s \\
\left|\zeta_{i}\right| & \leq 6 R \lambda \leq 12 R \sigma \sqrt{N}=3 s \sqrt{N}
\end{aligned}
$$

Hence, using the Bernstein inequality we get

$$
\begin{aligned}
& \operatorname{Prob}\left\{\sum_{i=1}^{N} \zeta_{i} \geq 8 R \sigma \sqrt{N}(\tau+1)\right\} \leq \operatorname{Prob}\left\{\sum_{i=1}^{N} \zeta_{i} \geq N r+(2 \tau+1) s \sqrt{N}\right\} \leq \\
& \leq \exp \left\{-\frac{(2 \tau+1)^{2} s^{2} N}{2 s^{2} N+\frac{2}{3} 3 s^{2} N(2 \tau+1)}\right\} \leq \exp \left\{-\frac{(2 \tau+1)^{2}}{2+2(2 \tau+1)}\right\} \leq e^{-\tau} .
\end{aligned}
$$

From (35) we also have

$$
\begin{aligned}
\mathbf{E}_{x_{i-1}}\left\{\varsigma_{i}\right\} & \leq \underbrace{4 \sigma^{2}}_{=: r}, \\
\left(\mathbf{E}_{x_{i-1}}\left\{\varsigma_{i}^{2}\right\}\right)^{1 / 2} & \leq 6 \lambda \sigma \leq 12 \sigma^{2} \sqrt{N}=: s, \\
\varsigma_{i} & \leq 9 \lambda^{2} \leq 36 \sigma^{2} N=4 s \sqrt{N} .
\end{aligned}
$$

Now, applying again the Bernstein inequality we get

$$
\begin{aligned}
\operatorname{Prob}\left\{\sum_{i=1}^{N} \varsigma_{i} \geq 16 N \sigma^{2}+24 N \sigma^{2} \tau\right\} & =\operatorname{Prob}\left\{\sum_{i=1}^{N} \varsigma_{i} \geq N r+(2 \tau+1) s \sqrt{N}\right\} \leq \\
& \leq \exp \left\{-\frac{(2 \tau+1) s^{2} N}{[2+2(2 \tau+1)] s^{2} N}\right\} \leq e^{-\tau} .
\end{aligned}
$$

Proofs of the bounds (32) and (33) in the case $M>\sigma \sqrt{N}$ and $\lambda=M+\sigma v$ follow the same lines.

A.2. Proof of Proposition 1. We first prove inequality (13). In view of (8), the optimality condition for (9) has the form

$$
\left\langle y_{i+1}+\psi^{\prime}\left(x_{i+1}\right)+\beta_{i}\left[\vartheta^{\prime}\left(x_{i+1}\right)-\vartheta^{\prime}\left(x_{i}\right)\right], z-x_{i+1}\right\rangle \geq 0, \quad \forall z \in X,
$$

or, equivalently,

$$
\begin{aligned}
\left\langle y_{i}+\psi^{\prime}\left(x_{i+1}\right), x_{i+1}-z\right\rangle & \leq \beta_{i}\left\langle\left[\vartheta^{\prime}\left(x_{i+1}\right)-\vartheta^{\prime}\left(x_{i}\right)\right], z-x_{i+1}\right\rangle=\left\langle\beta_{i} V_{x_{i}}^{\prime}\left(x_{i+1}\right), z-x_{i+1}\right\rangle= \\
& =\beta_{i}\left[V_{x_{i}}(z)-V_{x_{i+1}}(z)-V_{x_{i}}\left(x_{i+1}\right)\right], \quad \forall z \in X,
\end{aligned}
$$


where the last equality follows from the following remarkable identity (see, for example, [44]): for any $u, u^{\prime}$ and $w \in X$

$$
\left\langle V_{u}^{\prime}\left(u^{\prime}\right), w-u^{\prime}\right\rangle=V_{u}(w)-V_{u^{\prime}}(w)-V_{u}\left(u^{\prime}\right) .
$$

Since, by definition, $\xi_{i}=y_{i}-\nabla \phi\left(x_{i-1}\right)$ we get

$$
\begin{aligned}
\left\langle\nabla \phi\left(x_{i}\right), x_{i+1}-z\right\rangle+\left\langle\psi^{\prime}\left(x_{i+1}\right), x_{i+1}-z\right\rangle \leq & \beta_{i}\left[V_{x_{i}}(z)-V_{x_{i+1}}(z)-V_{x_{i}}\left(x_{i+1}\right)\right]- \\
& -\left\langle\xi_{i+1}, x_{i+1}-z\right\rangle .
\end{aligned}
$$

It follows from Lemma 1 and the condition $\beta_{i} \geq 2 L$ that

$$
F\left(x_{i+1}\right)-F(z) \leq \varepsilon_{i+1}(z) \leq\left\langle\nabla \phi\left(x_{i}\right), x_{i+1}-z\right\rangle+\left\langle\psi^{\prime}\left(x_{i+1}\right), x_{i+1}-z\right\rangle+\frac{\beta_{i}}{2} V_{x_{i}}\left(x_{i+1}\right) .
$$

Together with (37), this inequality implies

$$
\varepsilon_{i+1}(z) \leq \beta_{i}\left[V_{x_{i}}(z)-V_{x_{i+1}}(z)-\frac{1}{2} V_{x_{i}}\left(x_{i+1}\right)\right]-\left\langle\xi_{i+1}, x_{i+1}-z\right\rangle .
$$

On the other hand, due to the strong convexity of $V_{x}(\cdot)$ we have

$$
\begin{aligned}
\left\langle\xi_{i+1}, z-x_{i+1}\right\rangle-\frac{\beta_{i}}{2} V_{x_{i}}\left(x_{i+1}\right) & =\left\langle\xi_{i}, z-x_{i}\right\rangle+\left\langle\xi_{i+1}, x_{i}-x_{i+1}\right\rangle-\frac{\beta_{i}}{2} V_{x_{i}}\left(x_{i+1}\right) \\
& \leq\left\langle\xi_{i+1}, z-x_{i}\right\rangle+\frac{\left\|\xi_{i+1}\right\|_{*}^{2}}{\beta_{i}} .
\end{aligned}
$$

Combining these inequalities, we obtain

$$
F\left(x_{i+1}\right)-F(z) \leq \varepsilon_{i+1}(z) \leq \beta_{i}\left[V_{x_{i}}(z)-V_{x_{i+1}}(z)\right]-\left\langle\xi_{i+1}, x_{i}-z\right\rangle+\frac{\left\|\xi_{i+1}\right\|_{*}^{2}}{\beta_{i}}
$$

for all $z \in X$. Dividing (38) by $\beta_{i}$ and taking the sum over $i$ from 0 to $N-1$ we obtain (13).

We now prove the bound (14). Applying Lemma 6.1 of [1] with $z_{0}=x_{0}$ we get

$$
\forall z \in X, \quad \sum_{i=1}^{N} \beta_{i-1}^{-1}\left\langle\xi_{i}, z-z_{i-1}\right\rangle \leq V_{x_{0}}(z)+\frac{1}{2} \sum_{i=1}^{N} \beta_{i-1}^{-2}\left\|\xi_{i}\right\|_{*}^{2},
$$

where $z_{i}=\arg \min _{z \in X}\left\{\mu_{i-1}\left\langle\xi_{i}, z\right\rangle+V_{z_{i-1}}(z)\right\}$ depends only on $z_{0}, \xi_{1}, \ldots, \xi_{i}$. Further,

$$
\begin{aligned}
\sum_{i=1}^{N} \beta_{i-1}^{-1}\left\langle\xi_{i}, z-x_{i-1}\right\rangle & =\sum_{i=1}^{N} \beta_{i-1}^{-1}\left[\left\langle\xi_{i}, z_{i-1}-x_{i-1}\right\rangle+\left\langle\xi_{i}, z-z_{i-1}\right\rangle\right] \leq \\
& \leq V_{x_{0}}(z)+\sum_{i=1}^{N} \beta_{i-1}^{-1}\left\langle\xi_{i}, z_{i-1}-x_{i-1}\right\rangle+\frac{1}{2} \beta_{i-1}^{-2}\left\|\xi_{i}\right\|_{*}^{2} .
\end{aligned}
$$

Combining this inequality with (13), we get (14).

A.3. Proof of Corollary 1. Note that (15) is an immediate consequence of (13) and of the bounds for the moments of $\left\|\xi_{i}\right\|_{*}$ given in Lemma 2. Indeed, (29)(b) implies that, under the conditions of Corollary 1,

$$
\left|\mathbf{E}_{x_{i-1}}\left\{\left\langle\xi_{i}, z-x_{i-1}\right\rangle\right\}\right| \leq 2 D\left[(M+v \sigma)\left(\frac{\sigma}{\lambda}\right)^{2}+\frac{\sigma^{2}}{\lambda}\right] \leq \frac{2 D \sigma^{2}}{\lambda} \leq \frac{2 D \sigma}{\sqrt{N}}
$$


Further, due to $(29)(\mathrm{c})$,

$$
\mathbf{E}_{x_{i-1}}\left\{\left\|\xi_{i}\right\|_{*}^{2}\right\}^{1 / 2} \leq \sigma+(M+v \sigma) \frac{\sigma}{\lambda} \leq 2 \sigma .
$$

Taking the expectation of both sides of (13) and using the last two inequalities we get (15). The bound (17) is proved in a similar way, with the only difference that instead of inequality (13) we use (14).

A.4. Proof of Theorem 1. By virtue of part (i) of Lemma 3, under the condition $\tau \leq N / v^{2}$ we have that, with probability of at least $1-2 e^{-\tau}$,

$$
\begin{aligned}
\sum_{i=1}^{N}\left\langle\xi_{i}, z_{i-1}-x_{i-1}\right\rangle & \leq 16 R \max \{\sigma \sqrt{N \tau}, M \tau\}, \\
\sum_{i=1}^{N}\left\|\xi_{i}\right\|_{*}^{2} & \leq 40 \max \left\{N \sigma^{2}, M^{2} \tau\right\} .
\end{aligned}
$$

Plugging these bounds in (14) we obtain that, with probability at least $1-2 e^{-\tau}$, the following holds:

$$
\begin{aligned}
\bar{\beta} \sup _{z \in X} \varepsilon\left(x^{N}, z\right) & \leq 2 \bar{\beta} V_{x_{0}}(z)+\sum_{i=1}^{N}\left[\left\langle\xi_{i}, z_{i-1}-x_{i-1}\right\rangle+\frac{3}{2} \bar{\beta}^{-1}\left\|\xi_{i}\right\|_{*}^{2}\right] \leq \\
& \leq 2 \bar{\beta} R^{2} \Theta+16 R \max \{\sigma \sqrt{N \tau}, M \tau\}+60 \bar{\beta}^{-1} \max \left\{N \sigma^{2}, M^{2} \tau\right\} .
\end{aligned}
$$

Next, taking $\bar{\beta}=\max \left\{2 L, \frac{\sigma}{R} \sqrt{\frac{N}{\Theta}}\right\}$ we get

$$
\begin{aligned}
N\left[F\left(\widehat{x}_{N}\right)-F(z)\right] \leq & \max \left\{4 L R^{2} \Theta, 2 \sigma R \sqrt{N \Theta}\right\}+16 R \max \{\sigma \sqrt{N \tau}, M \tau\}+ \\
& +60 \max \left\{L R^{2} \tau / 2, \sigma R \sqrt{N \Theta}\right\} \leq \\
\leq & \max \left\{46 L R^{2} \tau, 4 L R^{2} \Theta, 62 \sigma R \sqrt{N \Theta}, 16 \sigma R \sqrt{N \tau}\right\}
\end{aligned}
$$

for $1 \leq \tau \leq N / v^{2}$. This implies (19).

A.5. Proof of Theorem 2. We act in the same way as in the proof of Theorem 1 with the only difference that instead of part (i) of Lemma 3 we use part (ii) of that lemma, which implies that if $N \geq v^{2}$ then with probability at least $1-2 e^{-\tau}$ the following inequalities hold:

$$
\begin{aligned}
\sum_{i=1}^{N}\left\langle\xi_{i}, z_{i-1}-x_{i-1}\right\rangle & \leq 8(1+\tau) R \max \{\sigma \sqrt{N}, M\} \\
\sum_{i=1}^{N}\left\|\xi_{i}\right\|_{*}^{2} & \leq 8(2+3 \tau) \max \left\{N \sigma^{2}, M^{2}\right\} .
\end{aligned}
$$

\section{A.6. Proof of Proposition 2. Define}

$$
\begin{aligned}
\rho_{N}(\tau ; \mu, \nu)=\nu^{-1} R^{2} \Theta & +16 R \max \{\sigma \sqrt{N \tau}, M \tau\}+ \\
& +20(\mu+\nu) \max \left\{N \sigma^{2}, M^{2} \tau\right\}+\mu^{-1} \sum_{i=1}^{N} V_{x_{i-1}}\left(x_{i}\right) .
\end{aligned}
$$

The proposition is a direct consequence of the following result.

Lemma 4 Define

$$
\begin{aligned}
\bar{\rho}_{N}(\tau)=\min _{\mu, \nu>0} \rho_{N}(\tau ; \mu, \nu)= & 4 R \sqrt{5 \Theta \max \left\{N \sigma^{2}, M^{2} \tau\right\}}+16 R \max \{\sigma \sqrt{N \tau}, M \tau\}+ \\
& +\min _{\mu>0}\left\{20 \mu \max \left\{N \sigma^{2}, M^{2} \tau\right\}+\mu^{-1} \sum_{i=1}^{N} V_{x_{i-1}}\left(x_{i}\right)\right\} .
\end{aligned}
$$

Then, for $0<\tau \leq N / v^{2}$ and $t \geq L$ the following inequalities hold

$\begin{array}{ll}\text { (a) } & \text { Prob }\left\{\epsilon_{N}(t)-\widehat{\epsilon}_{N}(t) \geq \bar{\rho}_{N}(\tau) / N\right\} \leq 2 \mathrm{e}^{-\tau}, \\ \text { (b) } & \operatorname{Prob}\left\{\widehat{\epsilon}_{N}(t)-\epsilon_{N}(t) \geq \bar{\rho}_{N}(\tau) / N\right\} \leq 2 \mathrm{e}^{-\tau} .\end{array}$ 
Proof of Lemma. Let us prove the first inequality in (42). Recall that $\xi_{i}=y_{i}-\nabla \phi\left(x_{i-1}\right)$, $i=1, \ldots, N$. Due to the strong convexity of $V_{x}(\cdot)$, for any $z \in X$ and $\mu>0$ we have

$$
\begin{aligned}
\left\langle\xi_{i}, z-x_{i}\right\rangle & =\left\langle\xi_{i}, z-x_{i-1}\right\rangle+\left\langle\xi_{i}, x_{i-1}-x_{i}\right\rangle \leq \\
& \leq\left\langle\xi_{i}, z-x_{i-1}\right\rangle+\frac{\mu}{2}\left\|\xi_{i}\right\|_{*}^{2}+\frac{1}{\mu} V_{x_{i-1}}\left(x_{i}\right) .
\end{aligned}
$$

Thus, for any $\nu>0$,

$$
\begin{aligned}
\sum_{i=1}^{N}\left\langle\xi_{i}, z-x_{i}\right\rangle & \leq \sum_{i=1}^{N}\left[\left\langle\xi_{i}, z-x_{i-1}\right\rangle+\frac{\mu}{2}\left\|\xi_{i}\right\|_{*}^{2}+\frac{1}{\mu} V_{x_{i-1}}\left(x_{i}\right)\right] \leq \\
& \leq \frac{1}{\nu} V_{x_{0}}(z)+\sum_{i=1}^{N}\left[\frac{\nu}{2}\left\|\xi_{i}\right\|_{*}^{2}+\left\langle\xi_{i}, z_{i-1}-x_{i-1}\right\rangle+\frac{1}{\mu} V_{x_{i-1}}\left(x_{i}\right)+\frac{\mu}{2}\left\|\xi_{i}\right\|_{*}^{2}\right]
\end{aligned}
$$

(to obtain the last inequality, we have used Lemma 6.1 from [1] with $z_{0}=x_{0}$ in the same way as in the proof of the Proposition 1). By Lemma 3 there is a set $\mathcal{A}_{N}$ of probability at least $1-2 \mathrm{e}^{-\tau}$ in the space of realizations $\omega^{N}$ such that, for all $\omega^{N} \in \mathcal{A}_{N}$,

$$
\sum_{i=1}^{N}\left\langle\xi_{i}, z_{i-1}-x_{i-1}\right\rangle \leq 16 R \max \{\sigma \sqrt{N \tau}, M \tau\} \text { and } \sum_{i=1}^{N}\left\|\xi_{i}\right\|_{*}^{2} \leq 40 \max \left\{N \sigma^{2}, M^{2} \tau\right\} .
$$

Recalling that $V_{x_{0}}(z) \leq R^{2} \Theta$, we conclude that $\sum_{i=1}^{N}\left\langle\xi_{i}, z-x_{i}\right\rangle \leq \rho_{N}(\tau ; \mu, \nu)$ for all $z \in X$ and all $\omega^{N} \in \mathcal{A}_{N}$. Therefore, for $\omega^{N} \in \mathcal{A}_{N}$ we have

$$
\epsilon_{N}(t)-\widehat{\epsilon}_{N}(t)=N^{-1} \sup _{z \in X} \sum_{i=1}^{N}\left\langle\xi_{i}, z-x_{i}\right\rangle \leq N^{-1} \min _{\mu, \nu \geq 0} \rho_{N}(\tau ; \mu, \nu)=N^{-1} \bar{\rho}_{N}(\tau),
$$

which proves the first inequality in (42). The proof of the second inequality in (42) is similar and therefore it is omitted.

A.7. Proof of Corollary 2. From the definition of $\epsilon_{N}(\cdot)$ we deduce that

$$
\bar{\beta} \sum_{i=1}^{N} V_{x_{i-1}}\left(x_{i}\right) \leq \epsilon_{N}(\bar{\beta})
$$

and we get (24) by taking $\mu=1 / \bar{\beta}$. On the other hand, one can check that for $\bar{\beta} \geq \max \left\{2 L, \frac{\sigma \sqrt{N}}{R \sqrt{\Theta}}\right\}$ the following inequalities hold:

$$
\begin{aligned}
\bar{\rho}(\tau) & \leq N \epsilon_{N}(\bar{\beta})+\max \left\{[(20+4 \sqrt{5}) \sqrt{\Theta}+16 \sqrt{\tau}] R \sigma \sqrt{N},(4 \sqrt{5 \Theta \tau}+26 \tau) L R^{2}\right\} \leq \\
& \leq N \epsilon_{N}(\bar{\beta})+C_{1} R \sigma \sqrt{N[\Theta \vee \tau]}+C_{2} L R^{2}[\Theta \vee \tau] .
\end{aligned}
$$

Finally, since $\widehat{\epsilon}_{N}(\bar{\beta}) \leq \epsilon_{N}(\bar{\beta})+\bar{\rho}(\tau) / N$ with probability at least $1-2 \mathrm{e}^{-\tau}$ (cf. (42)(b)) we have

$$
\Delta_{N}(\tau, \bar{\beta})=\widehat{\epsilon}_{N}(\bar{\beta})+\bar{\rho}(\tau) / N \leq \epsilon_{N}(\bar{\beta})+2 \bar{\rho}(\tau) / N
$$

with the same probability. This implies (25).

\section{A.8. Proof of Theorem 3.}

$\mathbf{1}^{o}$. We first show that for each $k=1, \ldots, m=m(N)$, the following is true. 
Fact $I_{k}$. There is a random event $\mathcal{B}_{k} \subseteq \Omega^{\otimes N}$ of probability at least $1-2 k e^{-\tau}$ such that for all $\omega^{N} \in \mathcal{B}_{k}$ the following inequalities hold:

(a) $\quad\left\|y_{k}-\bar{x}\left(y_{k}\right)\right\|^{2} \leq r_{k}^{2}=2^{-k} r_{0}^{2} \quad$ for some $\bar{x}\left(y_{k}\right) \in X_{*}$,

(b) $\quad F\left(y_{k}\right)-F_{*} \leq \frac{\kappa}{2} r_{k}^{2}=2^{-k-1} \kappa r_{0}^{2}$.

The proof of Fact $I_{k}$ is carried out by induction. Note that (43)(a) holds with probability 1 for $k=0$. Set $\mathcal{B}_{0}=\Omega^{\otimes N}$. Assume that (43)(a) holds for some $k \in\{0, \ldots, m-1\}$ with probability at least $1-2 k e^{-\tau}$, and let us show that then Fact $I_{k+1}$ is true.

Define $F_{*}^{k}=\min _{x \in X_{r_{k}}\left(y_{k}\right)} F(x)$ and let $X_{*}^{k}$ be the set of all minimizers of function $F$ on $X_{r_{k}}\left(y_{k}\right)$. By Theorem 1 and the definition of $N_{k}$ (cf. (27)), there is an event $\mathcal{A}_{k}$ of probability at least $1-2 e^{-\tau}$ such that for $\omega^{N} \in \mathcal{A}_{k}$ after the $(k+1)$-th stage of the algorithm we have

$$
\begin{aligned}
\frac{\kappa}{2}\left\|y_{k+1}-\bar{x}_{k}\left(y_{k+1}\right)\right\|^{2} \leq F\left(y_{k+1}\right)-F_{*}^{k} & \leq \max \left\{C_{1} \frac{L r_{k}^{2}[\tau \vee \Theta]}{N_{k+1}}, C_{2} \sigma r_{k} \sqrt{\frac{[\tau \vee \Theta]}{N_{k+1}}}\right\} \leq \\
& \leq \frac{\kappa}{4} r_{k}^{2}=\frac{\kappa}{2} r_{k+1}^{2},
\end{aligned}
$$

where $\bar{x}_{k}\left(y_{k+1}\right)$ is the projection of $y_{k+1}$ onto $X_{*}^{k}$. Set $\mathcal{B}_{k+1}=\mathcal{B}_{k} \cap \mathcal{A}_{k}$. Then

$$
\operatorname{Prob}\left\{\mathcal{B}_{k+1}\right\} \geq \operatorname{Prob}\left\{\mathcal{B}_{k}\right\}+\operatorname{Prob}\left\{\mathcal{A}_{k}\right\}-1 \geq 1-2(k+1) e^{-\tau} .
$$

In addition, due to the assumption of induction, on the set $\mathcal{B}_{k}$ (and, therefore, on $\mathcal{B}_{k+1}$ ) we have

$$
\left\|y_{k}-\bar{x}\left(y_{k}\right)\right\| \leq r_{k}
$$

i.e., the distance between $y_{k}$ and the set $X_{*}$ of global minimizers does not exceed $r_{k}$. Therefore, the set $X_{r_{k}}\left(y_{k}\right)$ has a non-empty intersection with $X_{*}$. Thus, $X_{*}^{k} \subseteq X_{*}$, the point $\bar{x}_{k}\left(y_{k+1}\right)$ is contained in $X_{*}$ and $F_{*}^{k}$ coincides with the optimal value $F_{*}$ of the initial problem. We conclude that

$$
\frac{\kappa}{2}\left\|y_{k+1}-\bar{x}\left(y_{k+1}\right)\right\|^{2} \leq F\left(y_{k+1}\right)-F_{*} \leq \frac{\kappa}{2} r_{k+1}^{2}=2^{-k} \kappa r_{0}^{2}
$$

for some $\bar{x}\left(y_{k+1}\right) \in X_{*}$.

$\mathbf{2}^{o}$. We now prove the theorem in the case $\bar{N}_{1} \geq 1$. This condition is equivalent to the fact that $\bar{N}_{k} \geq 1$ for all $k=1, \ldots, m(N)$, since $\bar{N}_{1} \leq \bar{N}_{2} \leq \cdots \leq \bar{N}_{m(N)}$ by construction. Assume that $\omega^{N} \in \mathcal{B}_{m(N)}$, so that (43) holds with $k=m(N)$. Since $\bar{N}_{1} \geq 1$ we have $N_{k} \leq 2 \bar{N}_{k}$. In addition, $\bar{N}_{k+1} \leq 2 \bar{N}_{k}$. Using these remarks and the definition of $m(N)$ we get

$$
N \leq \sum_{k=1}^{m(N)+1} N_{k} \leq 2 \sum_{k=1}^{m(N)+1} \bar{N}_{k} \leq 2 \sum_{k=1}^{m(N)} \bar{N}_{k}+4 \bar{N}_{m(N)} \leq 6 \sum_{k=1}^{m(N)} \bar{N}_{k} .
$$

Thus, using the definition of $\bar{N}_{k}$ (cf. (27)) we obtain

$$
\begin{aligned}
N & \leq 6 \sum_{k=1}^{m(N)} \max \left\{4 C_{1} \frac{L[\tau \vee \Theta]}{\kappa}, 16 C_{2} \frac{\sigma^{2}[\tau \vee \Theta]}{\kappa^{2} r_{k-1}^{2}}\right\} \leq \\
& \leq \underbrace{24 \sum_{k=1}^{\bar{k}-1} \frac{C_{1} L[\tau \vee \Theta]}{\kappa}}_{S_{1}}+\underbrace{96 \sum_{k=\bar{k}}^{m(N)} \frac{C_{2} \sigma^{2}[\tau \vee \Theta]}{\kappa^{2} r_{k-1}^{2}}}_{S_{2}}
\end{aligned}
$$


where

$$
\bar{k}=\min \left\{k: \frac{4 C_{2} \sigma^{2}}{\kappa r_{k-1}^{2}} \geq C_{1} L\right\} .
$$

Two cases are possible: $S_{1} \geq N / 2$ or $S_{2} \geq N / 2$. If $S_{1} \geq N / 2$, then

$$
\bar{k} \geq \frac{C^{\prime} \kappa N}{L[\tau \vee \Theta]},
$$

so that if $\omega^{N} \in \mathcal{B}_{m(N)}$ then

$$
F\left(\widehat{x}_{N}\right)-F_{*} \leq \frac{\kappa}{2} r_{m(N)}^{2} \leq \frac{\kappa}{2} r_{\bar{k}}^{2}=2^{-\bar{k}-1} \kappa r_{0}^{2} \leq C \kappa r_{0}^{2} \exp \left\{-\frac{C^{\prime} \kappa N}{L[\tau \vee \Theta]}\right\} .
$$

If $S_{2} \geq N / 2$ the following inequalities hold:

$$
\frac{\kappa^{2} r_{0}^{2} N}{\sigma^{2}[\tau \vee \Theta]} \leq \frac{C \kappa^{2} r_{0}^{2}}{\sigma^{2}[\tau \vee \Theta]} \sum_{k=\bar{k}}^{m(N)} 2^{k} \frac{\sigma^{2}[\tau \vee \Theta]}{\kappa^{2} r_{0}^{2}} \leq C^{\prime} 2^{m(N)-\bar{k}}
$$

Therefore, in this case for $\omega^{N} \in \mathcal{B}_{m(N)}$ we have

$$
F\left(\widehat{x}_{N}\right)-F_{*} \leq \frac{\kappa}{2} r_{m(N)}^{2}=\frac{\kappa}{2} r_{\bar{k}}^{2} 2^{-m(N)+\bar{k}} \leq \frac{\kappa}{2} r_{0}^{2} 2^{-m(N)+\bar{k}} \leq C \frac{\sigma^{2}[\tau \vee \Theta]}{\kappa N}
$$

$\mathbf{3}^{\circ}$. Finally, consider the case

$$
\bar{N}_{1}:=\max \left\{4 C_{1} \frac{L[\tau \vee \Theta]}{\kappa}, 16 C_{2} \frac{\sigma^{2}[\tau \vee \Theta]}{\kappa^{2} r_{0}^{2}}\right\}<1 .
$$

Let $k_{*} \geq 2$ be the smallest integer $k$ such that $\bar{N}_{k} \geq 1$. If $k_{*}>N / 4$ it is not difficult to see that $m(N) \geq N / 4$ and therefore for $\omega^{N} \in \mathcal{B}_{m(N)}$ we have

$$
F\left(y_{m(N)}\right)-F_{*} \leq \frac{\kappa}{2} r_{m(N)}^{2} \leq \frac{\kappa}{2} r_{0}^{2} 2^{-N / 4} .
$$

If $2 \leq k_{*} \leq N / 4$ we have the following chain of inequalities:

$$
3 \sum_{k=k_{*}}^{m(N)} \bar{N}_{k} \geq \bar{N}_{m(N)+1}+\sum_{k=k_{*}}^{m(N)} \bar{N}_{k}=\sum_{k=1}^{m(N)+1} \bar{N}_{k}-\sum_{k=1}^{k_{*}-1} \bar{N}_{k} \geq \sum_{k=1}^{m(N)+1} \bar{N}_{k}-N / 4 \geq N / 4,
$$

where the first inequality uses the fact that $\bar{N}_{m(N)+1} \leq 2 \bar{N}_{m(N)}$ and the last inequality follows from the definition of $m(N)$. Based on this remark and on the fact that $\bar{N}_{k} / 2^{k} \leq \bar{N}_{k_{*}} / 2^{k_{*}}$ for $k \geq k_{*}$ we obtain

$$
\frac{N}{12} \leq \sum_{k=k_{*}}^{m(N)} \bar{N}_{k} \leq \sum_{k=k_{*}}^{m(N)} 2^{k-k_{*}} \bar{N}_{k_{*}} \leq 2^{m(N)-k_{*}+2},
$$

where the last inequality follows by noticing that $\bar{N}_{k_{*}} \leq 2 \bar{N}_{k_{*}-1}<2$. Hence, taking into account (43)(b) we get that, for $\omega^{N} \in \mathcal{B}_{m(N)}$,

$$
F\left(\widehat{x}_{N}\right)-F_{*} \leq \kappa r_{k_{*}}^{2} 2^{-m(N)+k_{*}} \leq \kappa r_{0}^{2} 2^{-m(N)+k_{*}} \leq C \kappa r_{0}^{2} / N
$$

Combining this bound with (45), (46) and (48) we get (28). 


\section{References}

[1] Nemirovski, A., Juditsky, A., Lan, G., and Shapiro, A. Robust Stochastic Approximation Approach to Stochastic Programming, SIAM J. Optim., 2009, vol. 19, no. 4, pp. 1574-1609.

[2] Juditsky, A. and Nesterov, Y., Deterministic and Stochastic Primal-Dual Subgradient Algorithms for Uniformly Convex Minimization, Stoch. Syst., 2014, vol. 4, no. 1, pp. 44-80.

[3] Ghadimi, S. and Lan, G., Optimal Stochastic Approximation Algorithms for Strongly Convex Stochastic Composite Optimization I: A Generic Algorithmic Framework, SIAM J. Optim., 2012, vol. 22 , no. 4, pp. 1469-1492.

[4] Tukey, J.W., A Survey of Sampling from Contaminated Distributions, In: Olkin, I., et al. (eds.) Contributions to Probability and Statistics: Essays in Honor of Harold Hotelling, pp. 448-485. Stanford University Press, Palo Alto, 1960.

[5] Huber, P. J., Robust Estimation of a Location Parameter, Ann. Math. Statist., 1964, vol. 35, no. 1, pp. 73-101.

[6] Huber, P. J., Robust Statistics: A Review, Ann. Math. Statist., 1972, vol. 43, no. 4, pp. 10411067.

[7] Huber, P. J., Robust Statistics, New York: John Wiley and Sons, 1981.

[8] Martin, R. and Masreliez, C., Robust Estimation via Stochastic Approximation, IEEE Trans. Information Theory, 1975, vol. 21, no. 3, pp. 263-271.

[9] Polyak, B.T. and Tsypkin, Ya.Z., Adaptive Estimation Algorithms: Convergence, Optimality, Stability, Autom. Remote Control, 1979, vol. 40, no. 3, pp. 378-389.

[10] Polyak, B.T. and Tsypkin, Ya.Z., Robust Pseudogradient Adaptation Algorithms, Autom. Remote Control, 1981, vol. 41, no. 10, pp. 1404-1409.

[11] Polyak, B. and Tsypkin, J.Z., Robust Identification, Automatica, 1980, vol. 16, no. 1, pp. 53-63.

[12] Price, E. and Vandelinde, V., Robust Estimation Using the Robbins-Monro Stochastic Approximation Algorithm, IEEE Trans. Information Theory, 1979, vol. 25, no. 6, pp. 698-704.

[13] Stanković, S.S. and Kovačević, B.D., Analysis of Robust Stochastic Approximation Algorithms for Process Identification, Automatica, 1986, vol. 22, no. 4, pp. 483-488.

[14] Chen, H.-F., Guo, L., and Gao, A.J., Convergence and Robustness of the Robbins-Monro Algorithm Truncated at Randomly Varying Bounds, Stoch. Proc. Appl., 1987, vol. 27, pp. 217231.

[15] Chen, H.-F. and Gao, A.J., Robustness Analysis for Stochastic Approximation Algorithms, Stochast. Stochast. Rep., 1989, vol. 26, no. 1, pp. 3-20.

[16] Nazin, A.V., Polyak, B.T., and Tsybakov, A.B., Optimal and Robust Kernel Algorithms for Passive Stochastic Approximation, IEEE Trans. Information Theory, 1992, vol. 38, no. 5, pp. $1577-1583$.

[17] Tsypkin, Ya.Z., Osnovy Informatsionnoy Teorii Identifikatsii, Moscow: Nauka, 1984. (In Russian.) 
[18] Tsypkin, Ya.Z., Informatsionnaya Teoriya Identifikatsii, Moscow: Nauka, 1995. (In Russian.)

[19] Kwon, J., Lecué, G., and Lerasle, M., Median of Means Principle as a Divide-and-Conquer Procedure for Robustness, Sub-Sampling and Hyper-parameters Tuning, 2018, arXiv:1812.02435

[20] Chinot, G., Lecué, G., and Lerasle, M., Statistical Learning with Lipschitz and Convex Loss Functions, 2018, arXiv preprint arXiv:1810.01090.

[21] Lecué, G. and Lerasle, M. (2017). Robust machine learning by median-of-means: theory and practice, 2017, arXiv preprint arXiv:1711.10306v2. Annals of Stat., to appear.

[22] Lecué, G., Lerasle, M., and Mathieu, T. Robust Classification via MOM Minimization, 2018, arXiv preprint arXiv:1808.03106.

[23] Lerasle, M. and Oliveira, R. I., Robust empirical mean estimators, 2011, arXiv preprint arXiv:1112.3914.

[24] Lugosi, G. and Mendelson, S., Risk Minimization by Median-of-Means Tournaments, 2016, arXiv preprint arXiv:1608.00757.

[25] Lugosi, G. and Mendelson, S., Regularization, Sparse Recovery, and Median-of-Means Tournaments, 2017, arXiv preprint arXiv:1701.04112.

[26] Lugosi, G. and Mendelson, S., Near-Optimal Mean Estimators with Respect to General Norms, 2018, arXiv preprint arXiv:1806.06233.

[27] Hsu, D. and Sabato, S., Loss Minimization and Parameter Estimation with Heavy Tails, J. Machine Learning Research, 2016, vol. 17, no. 1, pp. 543-582.

[28] Bubeck, S., Cesa-Bianchi, N., and Lugosi, G., Bandits with Heavy Tail, IEEE Trans. Information Theory, 2013, vol. 59, no. 11, pp. 7711-7717.

[29] Devroye, L., Lerasle, M., Lugosi, G., and Oliveira, R.I., Sub-Gaussian Mean Estimators, Ann. Stat., 2016, vol. 44, no. 6, pp. 2695-2725.

[30] Nemirovskii, A.S. and Yudin, D.B., Slozhnost' zadach i effektivnost' metodov optimizatsii, Moscow: Nauka, 1979. Translated under the title Problem Complexity and Method Efficiency in Optimization, Chichester: Wiley, 1983.

[31] Lugosi, G. and Mendelson, S., Sub-Gaussian Estimators of the Mean of a Random Vector, Ann. Stat., 2019, vol. 47, no. 2, pp. 783-794.

[32] Catoni, O., Challenging the Empirical Mean and Empirical Variance: a Deviation Study, Ann. IHP: Probab. Stat., 2012, vol. 48, no. 4, pp. 1148-1185.

[33] Audibert, J.-Y. and Catoni, O., Robust Linear Least Squares Regression, Ann. Stat., 2011, vol. 39, no. 5, pp. 2766-2794.

[34] Minsker, S., Geometric Median and Robust Estimation in Banach Spaces, Bernoulli, 2015, vol. 21 , no. 4, pp. 2308-2335.

[35] Wei, X. and Minsker, S., Estimation of the Covariance Structure of Heavy-Tailed Distributions, In Advances in Neural Information Processing Systems, pp. 2859-2868, 2017. 
[36] Chen, Y., Su, L., and Xu, J., Distributed Statistical Machine Learning in Adversarial Settings: Byzantine Gradient Descent, Proceedings of the ACM on Measurement and Analysis of Computing Systems, vol. 1, iss. 2, article no. 44, 2017.

[37] Yin, D., Chen, Y., Ramchandran, K., and Bartlett, P., Byzantine-Robust Distributed Learning: Towards Optimal Statistical Rates, 2018, arXiv preprint arXiv:1803.01498.

[38] Cardot, H., Cénac, P., and Chaouch, M., Stochastic Approximation for Multivariate and Functional Median, In Proc. COMPSTAT'2010, pp. 421-428. Springer, 2010.

[39] Cardot, H., Cénac, P., and Godichon-Baggioni, A., Online Estimation of the Geometric Median in Hilbert Spaces: Nonasymptotic Confidence Balls, Ann. Stat., 2017, vol. 45, no. 2, pp. 591-614.

[40] Lan, G., An Optimal Method for Stochastic Composite Optimization, Math. Program., 2012, vol. 133, nos. 1-2, pp. 365-397.

[41] Necoara, I., Nesterov, Y., and Glineur, F., Linear Convergence of First Order Methods for Non-Strongly Convex Optimization, Math. Program., 2018, pp. 1-39.

[42] Juditsky, A. and Nemirovski, A., First Order Methods for Nonsmooth Convex Large-Scale Optimization, I: General Purpose Methods, In: S. Sra, S. Nowozin, and S. J. Wright (eds.), Optimization for Machine Learning, pp. 121-148. MIT Press, 2011.

[43] Freedman, D.A., On Tail Probabilities for Martingales, Ann. Probab., 1975, vol. 3, no. 1, pp. $100-118$.

[44] Chen, G. and Teboulle, M., Convergence Analysis of a Proximal-Like Minimization Algorithm Using Bregman Functions, SIAM J. Optim., 1993, vol. 3, no. 3, pp. 538-543. 